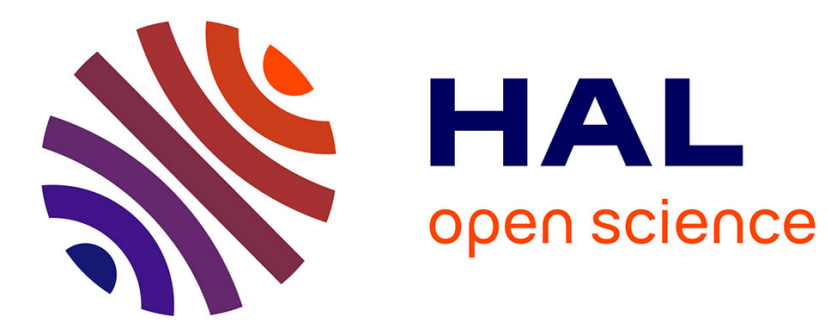

\title{
Graph Structuring of Skeleton Object for Its High-Level Exploitation
}

Rabaa Youssef, Anis Kacem, Sylvie Sevestre-Ghalila, Christine Chappard

\section{To cite this version:}

Rabaa Youssef, Anis Kacem, Sylvie Sevestre-Ghalila, Christine Chappard. Graph Structuring of Skeleton Object for Its High-Level Exploitation. 12th International Conference on Image Analysis and Recognition (ICIAR 2015), Jul 2015, Niagara Falls, ON, Canada. pp.419 - 426, 10.1007/978-3319-20801-5_46. hal-01436778

\section{HAL Id: hal-01436778 https://hal.science/hal-01436778}

Submitted on 18 Jan 2017

HAL is a multi-disciplinary open access archive for the deposit and dissemination of scientific research documents, whether they are published or not. The documents may come from teaching and research institutions in France or abroad, or from public or private research centers.
L'archive ouverte pluridisciplinaire HAL, est destinée au dépôt et à la diffusion de documents scientifiques de niveau recherche, publiés ou non, émanant des établissements d'enseignement et de recherche français ou étrangers, des laboratoires publics ou privés. 


\title{
Graph structuring of skeleton object for its high-level exploitation
}

\author{
Rabaa Youssef ${ }^{1,2}$, Anis Kacem ${ }^{2}$, Sylvie Sevestre-Ghalila ${ }^{2}$, Christine Chappard ${ }^{3}$ \\ 1. COSIM, University of Carthage, Cité Technologique des Communications, Tunisia \\ 2. CEA-LIST CEA-LinkLab, Cité Technologique des Communications, Tunisia \\ 3. B2OA, UMR CNRS 7052, Université Paris Diderot, PRES Sorbonne Paris Cité, \\ Paris, France
}

\begin{abstract}
Skeletonization is a morphological operation that summarizes an object by its median lines while preserving the initial image topology. It provides features used in biometric for the matching process, as well as medical imaging for quantification of the bone microarchitecture. We develop a solution for the extraction of structural and morphometric features useful in biometric, character recognition and medical imaging. It aims at storing object descriptors in a re-usable and hierarchical format. We propose graph data structures to identify skeleton nodes and branches, link them and store their corresponding features. This graph structure allows us to generate CSV files for high level analysis and to propose a pruning method that removes spurious branches regarding their length and mean gray level. We illustrate manipulations of the skeleton graph structure on medical image dedicated to bone microarchitecture characterization.
\end{abstract}

\section{Introduction}

Skeletonization is used in various applications such as biometrics 112, medical imaging [3445] and character recognition [6] since it provides features that enables user to access high-level analysis of the image objects. In fact, object matching methods based on skeleton features are used in biometric identification through minutiae comparison of hand vein 1 or digital fingerprint 2, in bronchial airway trees monitoring [5], in symbols identification [7] and in character recognition [6]. In addition, classification methods using morphometric features extracted from skeleton helps diagnose osteoporosis 4 and osteoarthritis 3 diseases.

Graph-based representation of the skeleton is widely investigated for matching issues since the correspondence between skeleton branches with graph edges and nodes with its vertices is natural and intuitive. The proposed solutions in the literature are based on shock graphs $[7$ or attributed relational graphs [9. These representations based on adjacency matrix can only consider acyclic graphs which restricts the application domains.

Our contribution is to develop a solution for the exploitation of skeleton features in binary and grayscale domain and to save them in a re-usable format easily 
manipulated. We propose to construct a graph able to represent any skeleton and to store its structural and morphometric features. To this purpose, we identify skeleton with multigraph [10] where multiple edges between nodes and emergence of mass of junctions are permitted. We store the skeleton structural and morphometric features in data structures that link graph vertices to their adjacent edges. This solution allows us to manipulate the skeleton information and to implement a pruning procedure that takes into account not only segments lengths but also their average gray values in order to decide which branches are spurious and thus, delete them. The use of this contribution is illustrated in the context of medical application that cover almost all usable features.

This work is composed of five sections. The first one presents skeleton features for medical application which covers biometric and character recognition structural features. The second section describes the used topological definitions for the graph construction. The third section is devoted to the graph data structures proposed in this work. The fourth section is dedicated to the implemented pruning and the manipulation of the structured graph skeleton on image of subchondral bone in the tibial knee with the aim of characterizing its microarchitecture.

\section{Skeleton features in medical imaging}

Concerning the characterization of bone microarchitecture, studies has shown that bone changes from a healthy person to a person with osteoporosis [4] or osteoarthritis 3]. These changes are quantified using morphometric parameters of bone microarchitecture such as the number of pixels of the skeleton, the halfwidth and the length of trabeculae, the number of trabeculae and the number of nodes and ends. These morphometric parameters covers also structural descriptors (detection of nodes and extremities) used in biometric and character recognition applications [1] [2] 6]. We notice that skeletonization methods used to extract features for applications cited above are binary or gray. Consequently, we choose to make this feature extraction tool applicable on both types of skeleton. Furthermore, we notice that skeleton gray levels are not actually exploited. We propose to consider in the extracted features the segment mean gray level which is useful for the pruning of insignificant skeleton branches.

\section{Identification of topological configurations}

First of all, we need to identify the topological nature of each skeleton point in order to position it in the graph. A skeleton is formed of ridge, junction and end pixels. A ridge is a skeleton pixel placed on a crest line that splits the local background in 24 -connected components. A junction splits the local background to 3 or 4 -connected components and an end is a skeleton pixel with a unique skeleton neighbor. 


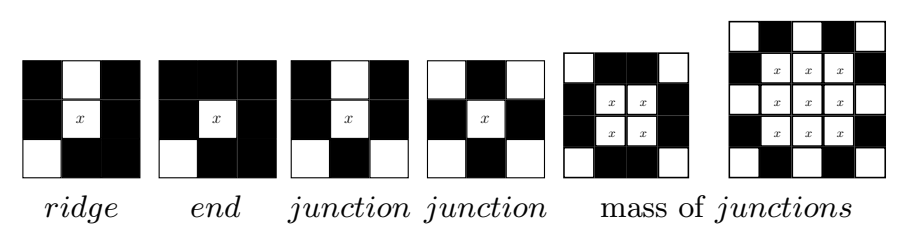

Fig. 1. Topological configurations of skeleton pixels.

Our graph proposal is designed to accept as input any type of skeleton. To do so, it is important to identify critical situations that a conventional graph cannot handle. Indeed, a skeleton could have a mass of junctions that cannot be thinned. In Figure 1, pixels noted $x$ and identified as mass of junctions divide the background in two 4-connected components according to their 8-neighbors. However, these pixels belong to a mass of nodes that should be entirely considered as a unique junction. In the literature, an erosion at nodes is performed before the construction of adjacency matrix in order to eliminate such configurations and to avoid a cyclic graph. A decomposition of the possible mass junctions in $3 \times 3$ configurations (with rotations) is made for our proposal in order to identify these pixels when processing the skeleton for graph construction. Once the ambiguous configurations identified, we can proceed with the construction of graph data structures.

\section{Graph construction}

Since we intend to conceive a graph that takes into account any kind of skeleton, simple graphs constructed using an adjacency matrix are not appropriate. A graph with multiple edges (multigraph) that manages mass of junctions and stores other morphometric features of skeleton branches is chosen in this work. We detail in the next the data structures used to construct the proposed multigraph and the linking step that permits to connect graph vertices and edges.

\subsection{Data structures for the graph construction}

First, we define in Table1 Point data structure that differentiates end, junction and ridge pixels and establish a primary link between current pixel and its direct skeleton neighbors.

Table 1. Data structure: Point

\begin{tabular}{|c|c|c|c|}
\hline Structure Point & \multicolumn{3}{|c|}{ Description } \\
\hline identifier & \multicolumn{3}{|c|}{ pixel index in the image (unique) } \\
\hline $\begin{array}{c}\text { Flag } \\
\text { Neighbors }\end{array}$ & \begin{tabular}{|c|}
$(1)$ : end \\
only one 8-connected neighbor
\end{tabular} & \begin{tabular}{|c|}
$(2):$ ridge \\
Table of size $[2 . .4]$
\end{tabular} & $\begin{array}{c}(3): \text { junction } \\
\text { Table of size }[3 . .8]\end{array}$ \\
\hline
\end{tabular}


Having defined the point structure, we can define the Segment structure that corresponds to a graph edge. Intuitively, a segment has two extremities. There are 3 types of segments. The first one has two free extremities. The second has its extremities identified as junctions and the third one has a free end and a junction at its respective extremities. Therefore, we need to identify the segment extremities in the data structure in order to point out the segment type. The data structure segment is defined by the attributes listed in Table 2 .

Table 2. Data structure: Segment

\begin{tabular}{|c|c|}
\hline Structure Segment & Description \\
\hline Label & segment identifier \\
\hline Head & Table of point indentifiers for the segment head (maximum size is 3 ). \\
\hline Next to head & Index of the point that follows the head in the segment \\
\hline Tail & Table of point indentifiers for the segment tail (maximum size is 3 ) \\
\hline Next to tail & Index of the point that follows the tail in the segment \\
\hline Stack & stack containing segment pixels (index of ridge points) \\
\hline Segment features & Number of pixels, Euclidean length, Mean gray level, Half width \\
\hline
\end{tabular}

Tail and head attributes correspond to segment extremities. These attributes are useful to establish links between graph edges. Since our solution is intended to be general, covering all types of skeleton graph, we consider segments having multiple heads and tails as illustrated by Figure 2.(a). Hence, we choose for the head attribute (respectively tail) an array of points of size 3 .

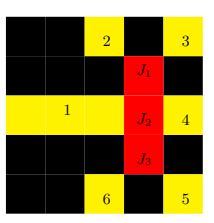

(a): 3 junctions in red for the "head" of segment 1

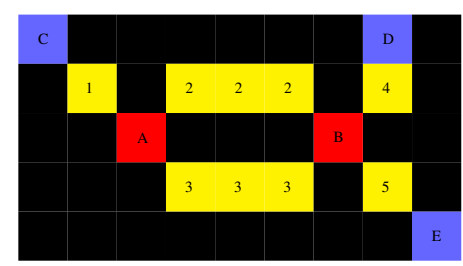

(b): Multiple segments between two junctions

Fig. 2. Particular situations for segment structure. In blue: segment end. In red: segment head (or tail) labeled as junction. In yellow: segment ridge point.

Furthermore, and unlike graphs based on adjacency matrix that cannot handle loops, we get our solution to consider a general case that defines more than one segment between two nodes. Consequently, we add two other attributes to the data structure, which are "Next to head" and "Next to queue" in order to distinguish the multiple segments appearing between two junction pixels. Such a case is illustrated by Figure 2. (b). 


\subsection{Linking step for segment structure}

This module enables the implementation to fill point data structures and then, create segment structures by browsing the skeleton. It proceeds by first searching for graph nodes (junctions or ends) and second, by monitoring the ridge pixels of the same segment structure. Using the "neighbor" attribute stored in the point structure, it is possible to follow the segment and fill its remaining attributes. A stop condition of filling a segment is the detection of an end or other junction. This means that the linking step reached the end of the segment, can finally calculate its features and move to the next segment via the "tail" attribute. Figure 3 illustrates an example of segments attributes obtained for the binary skeleton sample of Figure 2(b).

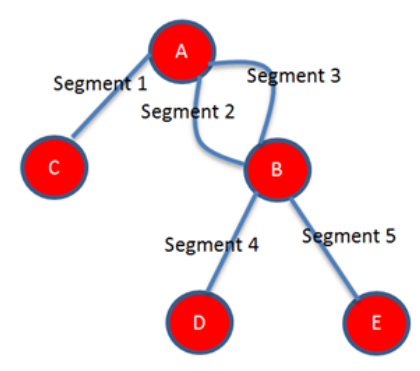

\begin{tabular}{|c|c|c|c|c|c|}
\hline Attributes & Seg 1 & Seg 2 & Seg 3 & Seg 4 & Seg 5 \\
\hline Label & 1 & 2 & 3 & 4 & 5 \\
\hline Head & C & A & A & B & B \\
\hline Next to head & 1 & 2 & 6 & 5 & 9 \\
\hline Tail & $\mathrm{A}$ & $\mathrm{B}$ & $\mathrm{B}$ & $\mathrm{D}$ & $\mathrm{E}$ \\
\hline Next to tail & 1 & 4 & 8 & 5 & 9 \\
\hline Stack & 1 & $2,3,4$ & $6,7,8$ & 5 & 9 \\
\hline $\begin{array}{c}\text { Number of } \\
\text { pixels }\end{array}$ & 3 & 5 & 5 & 3 & 3 \\
\hline $\begin{array}{c}\text { Euclidean } \\
\text { length }\end{array}$ & $2 \cdot \sqrt{2}$ & $2+2 \cdot \sqrt{2}$ & $2+2 \cdot \sqrt{2}$ & $1+\sqrt{2}$ & $2 \cdot \sqrt{2}$ \\
\hline $\begin{array}{c}\text { Average gray } \\
\text { level }\end{array}$ & 255 & 255 & 255 & 255 & 255 \\
\hline
\end{tabular}

Fig. 3. Segments data structures for image from Figure 2(b).

Finally, the flow chart of Figure 4 resumes the complete processing stages for the extraction of skeleton features. The graph construction is applicable to gray or binary skeleton resulting from any method of the literature.

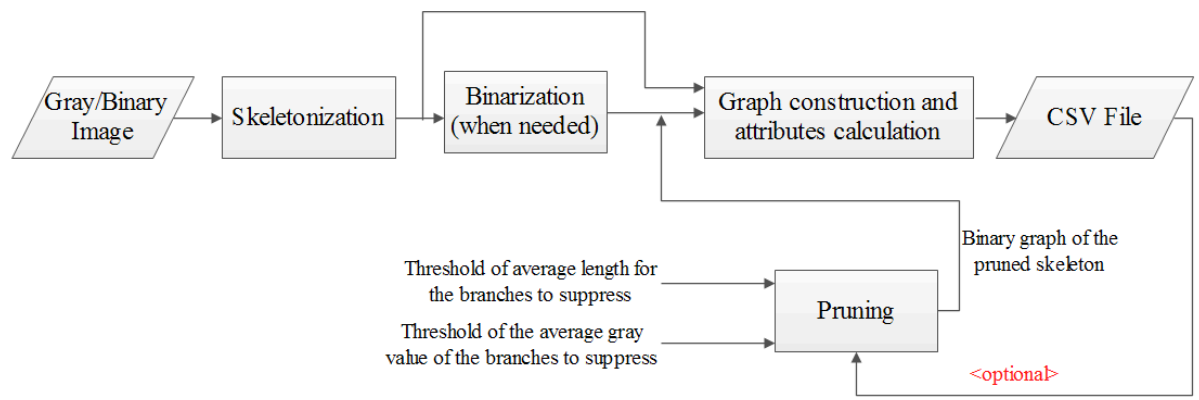

Fig. 4. Flow chart of features extraction tool. 


\section{$5 \quad$ Pruning as an application of graph structuring}

Skeletonization methods, and especially homotopic thinning are often subject to the emergence of spurious branches. For better reliability of results and pertinence of analysis, a pruning step is performed to eliminate these branches. In some applications, it is the orientation of the segment that indicates its "significance". However, orientation cannot be pertinent for microarchitecture quantification since trabeculae moves in various directions. Through experiments, we notice that the average gray level of spurious branches is informative and generally smaller than the average gray level of significant ones. We propose to implement a pruning procedure based on our graph construction that takes into account not only the length but also the mean gray value of segments to suppress noisy branches. In fact, according to the resulting skeletons of Figure 5 and their respective features, we can establish an upper threshold for segment lengths and gray values at a fixed percentile (50\% in this case). These thresholds adequately adjusted permit to detect spurious branches and delete them in both cases.

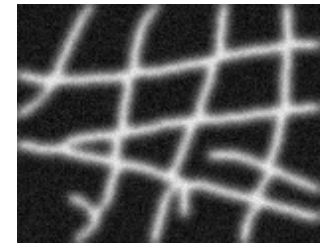

Original image

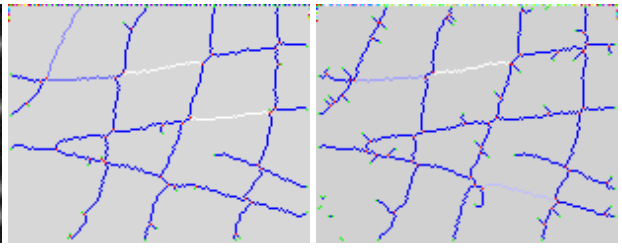

Skeleton 2

\begin{tabular}{c|c|c}
\hline & Features for segments with free ends & Percentile(50\%) \\
\hline \multirow{2}{*}{ Skeleton 1 } & Pixelwise length & 5 \\
& Mean gray level & 183,38 \\
\hline \multirow{2}{*}{ Skeleton 2 } & Pixelwise length & 6 \\
& Mean gray level & 120,72 \\
\hline
\end{tabular}

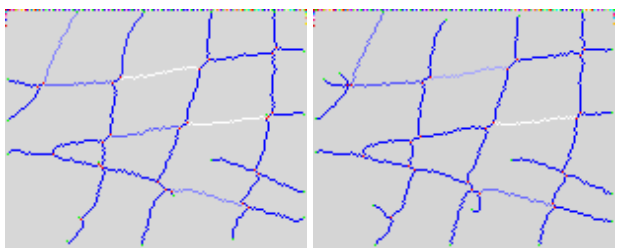

Pruning 1

Pruning 2

Fig. 5. Results of gray skeletonization using methods published in [11 and in [12. Color intensity (white to blue) reflects segments lengths.

An application of such process is performed on high resolution peripheral computed tomography (HR-pQCT) slice image from Voxelo project 1 as illus-

\footnotetext{
${ }^{1}$ ANR Voxelo TECS-0018
} 
trated in Figure6. We note respectively Nb.S, Nb.N, Nb.E, Nb.seg the number of skeleton pixels, nodes, ends and segments, and $\overline{\text { Nb.pix seg }}, \overline{\text { Length of seg }}$, Gray level of seg the mean pixelwise length, euclidian length an gray level of segments. We observe in the update of skeleton features a gain in pertinence after pruning spurious branches while preserving significant trabeculae.

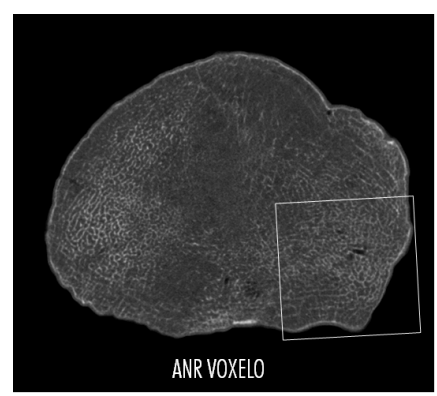

Box: Region of Interest

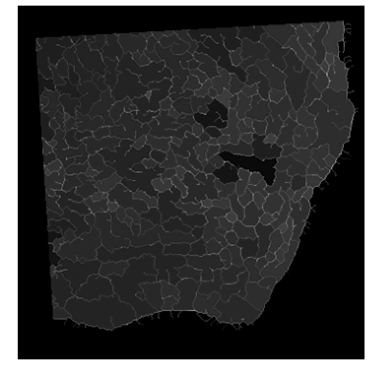

Gray skeleton

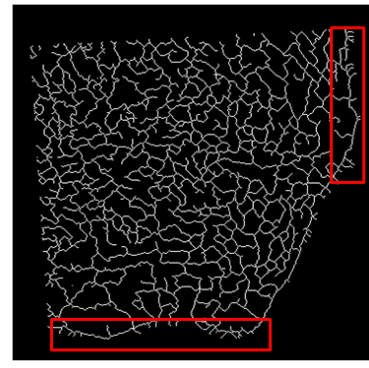

Before pruning

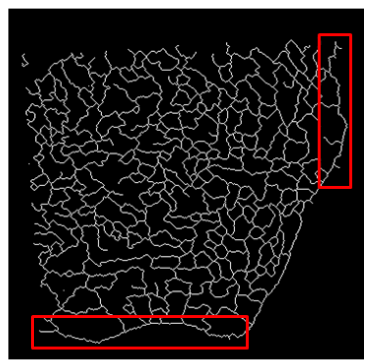

After pruning

\begin{tabular}{|c|c|c|c|c|c|c|c|c|}
\hline & Bone surfac & $|N b . S|$ & Nb.N & Nb.E & Nb.seg & b.pix s & gth of & level of seg \\
\hline Before pruning & 93529 & 10734 & 650 & 294 & 1168 & 9,85 & 9,34 & 93,97 \\
\hline After pruning & 93529 & 9265 & 432 & 48 & 692 & 13,67 & 13,65 & 94,89 \\
\hline
\end{tabular}

Fig. 6. Skeleton pruning of the trabecular microarchitecture in a slice image of suchondral bone in tibial knee: Gain in relevance of features.

\section{Conclusion}

The proposed graph construction applies to binary and gray skeletons and facilitate a high level manipulation of the skeleton via the structural and morphometric features it stores. The analysis of the trabecular microarchitecture aiming to differentiate normal bone from osteoarthritis is an example of skeleton graph structure usefulness. The pruning procedure exploiting graph and skeleton features eliminates efficiently spurious branches according to thresholds of the 
mean gray level and length of segments. This high level skeleton manipulation can be used in biometric and character recognition applications in the matching process. Our perspective is to create a GUI for easy handling of the graph: tracking lines, measuring distance between nodes/edges, updating interactively the features. In addition, other features such as branches orientation could be easily added to the data structure segment in order to monitor skeleton lines and add another optional threshold to the pruning.

\section{Acknowledgments}

This work is financed by ANR Voxelo TECS-0018.

\section{References}

1. Zhu, X. and Huang, D.: Hand Dorsal Vein Recognition Based on Hierarchically Structured Texture and Geometry Features. Biometric Recognition, 7701:157-164 (2012).

2. Zhao, F. and Tang, X.: Preprocessing and postprocessing for skeleton-based fingerprint minutiae extraction. Pattern Recognition, 40(4):1270-1281 (2007).

3. Chappard, C., Peyrin, F., Bonnassie, A., Lemineur, G., Brunet-Imbault, B., Lespessailles, E. and Benhamou, C.L.: Subchondral bone micro-architectural alterations in osteoarthritis: a synchrotron micro-computed tomography study. Osteoarthritis and Cartilage, 14(3):215-223 (2006).

4. Sevestre-Ghalila, S., Benazza Benyahia, A., Ricordeau, A., Mellouli, N. and Chappard, C.: Texture Image Analysis for Osteoporosis Detection with Morphological Tools. In: Medical Image Computing and Computer-Assisted Intervention MICCAI, 3216:87-94 (2004).

5. Tschirren, J., Palàgyi, K., Reinhardt, J.M., Hoffman, E.A. and Sonka, M.: Shock Segmentation, Skeletonization, and Branchpoint Matching A Fully Automated Quantitative Evaluation of Human Intrathoracic Airway Trees. In: Medical Image Computing and Computer-Assisted Intervention MICCAI, 2489:12-19 (2002).

6. Wshah, S., Zhixin, S. and Govindaraju, V.: Segmentation of Arabic Handwriting Based on both Contour and Skeleton Segmentation. In: 10th International Conference on Document Analysis and Recognition -ICDAR, 793-797 (2009).

7. Siddiqi, K., Shokoufandeh, A., Dickenson, S.J. and Zucker, S.W.: Shock graphs and shape matching. In: 6th International Conference on Computer Vision, 222229 (1998).

8. Xiang, B. and Latecki, L.J.: Path Similarity Skeleton Graph Matching. IEEE Transactions on Pattern Analysis and Machine Intelligence, 30(7):1282-1292 (2008).

9. Di Ruberto, C.: Recognition of shapes by attributed skeletal graphs. Pattern Recognition, 37(1):21-31 (2004).

10. Gross, J.L. and Yellen, J.: Graph Theory and Its Applications, Second Edition (Discrete Mathematics and Its Applications). Chapman \& Hall/CRC (2005).

11. Youssef, R., Sevestre-Ghalila, S. and Ricordeau, A.: Statistical control of thinning algorithm with implementation based on hierarchical queues. In: 6th International Conference of Soft Computing and Pattern Recognition -SoCPaR, 365-370 (2014).

12. Couprie, M., Bezerra, F.N. and Bertrand, G.: Grayscale image processing using topological operators. SPIE Vision Geometry VIII., 3811:261-272 (1999). 